\title{
Photosystem I: a search for green plant trimers $\dagger$
}

\author{
Roman Kouřil, Niels van Oosterwijk, Alevtyna E. Yakushevskał and Egbert J. Boekema* \\ Department of Biophysical Chemistry, Groningen Biomolecular Sciences and Biotechnology \\ Institute, University of Groningen, Nijenborgh 4, 9747, AG, Groningen, The Netherlands. \\ E-mail: e.j.boekema@rug.nl; Fax: +3150 3634800; Tel: +31 503634225
}

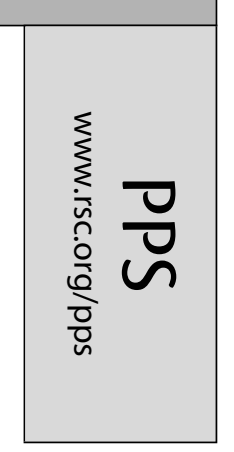

Received 20th April 2005, Accepted 23rd June 2005

First published as an Advance Article on the web 25th July 2005

\begin{abstract}
Recent blue-native gel electrophoresis studies gave evidence for the existence of dimeric and trimeric PSI complexes in green plants. We used single particle electron microscopy to investigate all the larger particles from the thylakoid membrane of pea (Pisum sativum var. Charmette). Peak fractions with monomeric, dimeric and trimeric Photosystem I were obtained after solubilization with digitonin and size-exclusion chromatography. The analysis showed that only a few percent of dimers and trimers were present. In the best resolved trimers some of the monomers were oriented upside down. Many classes were fuzzy, indicating a non-specific or flexible orientation. From these results we conclude that the green plant PSI is monomeric within the green plant membrane.
\end{abstract}

\section{Introduction}

Photosystem I (PSI) is a multisubunit protein complex embedded in the non-appressed thylakoid membranes of cyanobacteria, green algae and plants ${ }^{1}$ and currently, the X-ray structures of cyanobacterial and plant PSI are known at 2.5 and $4.4 \AA$ resolution, respectively., ${ }^{2,3}$ Although the two overall structures show similar features, there are a few differences, which make plant PSI rather unique. First, the presence of the PsaG subunit in plant PSI enables anchoring of a belt of four transmembrane Lhca1-4 light-harvesting proteins (LHCI) from the PsaF/J side to the PSI core complex., Second, the plant PSI differs from cyanobacterial PSI by a presence of the PsaH subunit, which was found to be essential for a docking of LHCII, a light harvesting complex of Photosystem II (PSII), under state transitions. ${ }^{5,6}$ The PsaH subunit is bound at the periphery of the core complex next to the PsaL subunit.

Cyanobacterial PSI has the ability to form native trimers in the photosynthetic membrane ${ }^{7}$ and the PsaL subunit is involved in the trimerization. ${ }^{8}$ Phosphatidylglycerol also plays an indispensable role in the PsaL-mediated assembly of the PSI trimers. ${ }^{9}$ In plants, there is no direct evidence for the presence of native PSI trimers in the thylakoid membrane. One strong argument against trimers is the presence of the PsaH, which surrounds and modifies the PsaL. Therefore plant PSI is an unable to form trimers in the same way as in cyanobacteria ${ }^{10}$ and a monomeric PSI is expected to be present in the thylakoid membrane of green algae and plants in vivo.

However, recent biochemical experiments with digitonin solubilized thylakoid membranes and subsequent $2 \mathrm{D}$ blue native polyacrylamide gel electrophoresis showed the presence of two PSI complexes with apparent molecular weights of about 1060 and $1600 \mathrm{kDa}$, which were assigned as dimers and trimers of plant PSI. ${ }^{11}$ However, the authors could not rule out the possibility of artificial association of PSI. On the other hand there is increasing evidence that the detergent digitonin has a specific ability to preserve the association of supercomplex components, in contrast to the commonly used detergents $\alpha$ or $\beta$-D-dodecyl maltoside. Recently, the first supercomplex from plant mitochondria, consisting of complexes I and III of the

$\dagger$ Dedicated to Professor James Barber on the occasion of his 65th birthday.

$\ddagger$ Present address: Molecular Cell Biology, Faculty of Biology, Utrecht University, Utrecht, The Netherlands. respiratory chain and purified after digitonin solubilization, was characterized. ${ }^{12}$

This raises the question again whether plant PSI dimers and/or trimers exist, but with the monomers associated in a way differing from that in the cyanobacteria. One possibility would be to analyse PSI supercomplexes within the intact membrane, in a way as has been performed for spinach grana membrane fragments enriched in Photosystem II. ${ }^{13}$ In principle, such an electron microscopy (EM) analysis could even be performed on osmotically shocked chloroplasts without a detergent solubilization step, because PSI protrudes from the stroma lamella. However, in negatively stained specimens the PSI particles are barely visible, in contrast to ATP synthase and Rubisco, which protrude farther from the membrane surface. ${ }^{1}$ Thus, the other possibility, to analyse detergent solubilized particles, remains as the alternative. Previously, experiments have been performed with particles purified after solubilization with $\beta$ - or $\alpha$-D-dodecyl maltoside. EM analysis revealed that solubilization of spinach thylakoid membranes using $\alpha$-D-dodecyl maltoside can lead to either dimerization or trimerization of PSI. ${ }^{4}$ However, image analysis revealed that this is an artificial association, because PSI monomers associate with different handedness (up and down-oriented), which is certainly not reflecting the membrane situation. A similar type of dimer was found for PSI from Arabidopsis thaliana (E. J. Boekema, unpublished results). In addition, the spinach PSI monomers showed an unusual strong tendency to associate into two-dimensional sheets after sucrose density gradient centrifugation (A. E. Yakushevska, unpublished results). The various types of particles and associates found previously have been compiled in Fig. 1.

In this work, we have addressed the trimer question once again, but now with the detergent digitonin. We have performed an EM analysis of PSI complexes purified after solubilization of pea thylakoid membranes using digitonin, and revealed presence of monomers, dimers and trimers of PSI in EM micrographs. Image analysis of single particle projections revealed a novel type of interaction between PSI monomers, which was mediated by LHCI. However, the best resolved part of our data set indicates that this association of monomers is artificial. The nature of the rest of our data set is not clear. A small set of trimers might reflect the situation in the membrane, with all monomers in the same orientation with respect to the membrane plane. Nevertheless, from these experiments we conclude that the plant PSI is monomeric. 


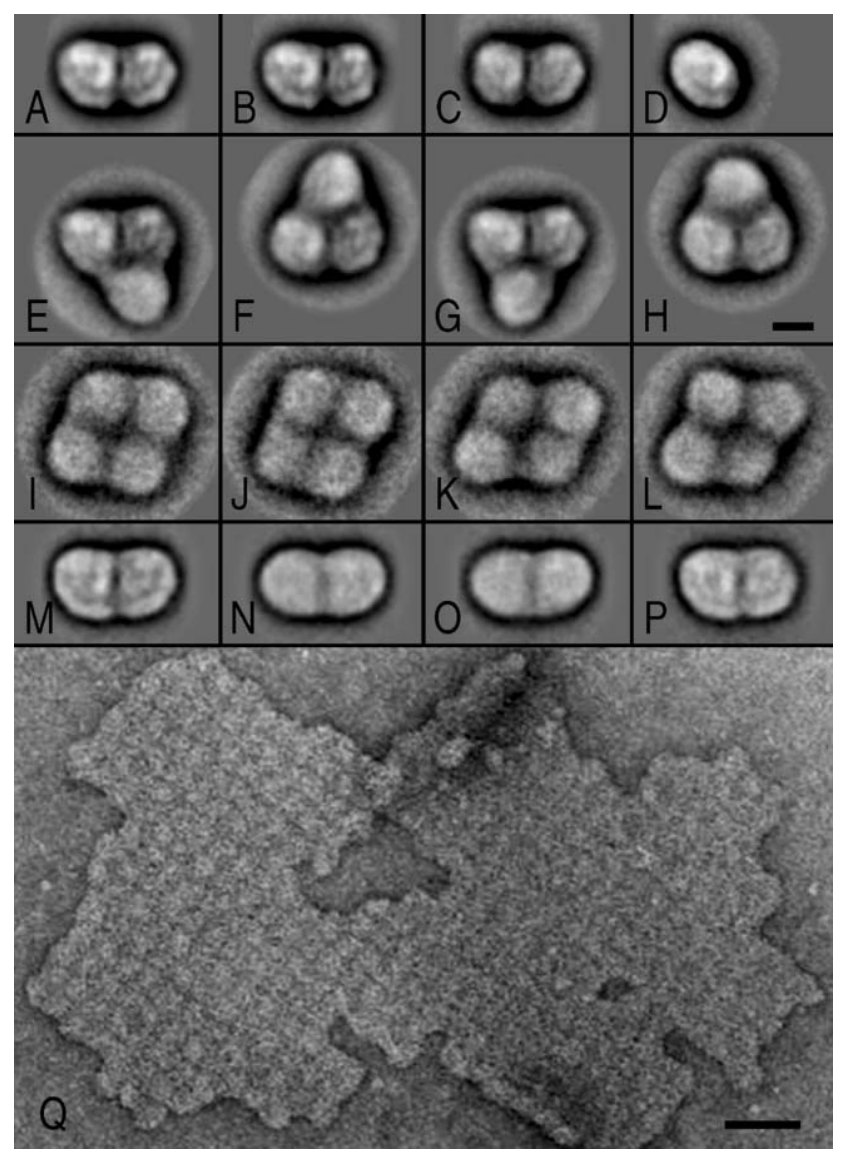

Fig. 1 (A)-(L) Monomeric and multimeric PSI complexes from spinach thylakoids. (A) artificial dimers consisting of two intact upand down-oriented monomers. (B), (C) Similar dimers, but with a protein mass missing at the PsaK side at the right and left monomer, respectively. (D) Monomeric PSI lacking substantial mass at and around the PsaL/H subunits. (E)-(H) Artificial trimers; they are positioned in such a way that two of the monomers are positioned as in the dimers. A next monomer is associated at different positions. (I)-(L) Artificial PSI tetramers, with a variable overall shape. Due to the lower number of particles summed, inner features are obscured by the higher noise level (E. J. Boekema, unpublished results). (M)-(P) Artificial dimers consisting of two intact up- and down-oriented monomers purified from Arabidopsis thaliana. Classes $\mathrm{N}$ and $\mathrm{O}$ look fuzzy, indicating that the association is not very specific but variable. (Q) PSI two-dimensionally aggregated PSI complexes, obtained without further treatment after sucrose density gradient centrifugation after solubilization of spinach thylakoid membranes under stacking conditions with $\beta$-D-dodecyl maltoside at a detergent to Chl molar ratio of $1: 10$. The space bar in $(\mathrm{A})-(\mathrm{P})$ equals $10 \mathrm{~nm}$, the bar in $(\mathrm{Q})$ is $50 \mathrm{~nm}$.

\section{Experimental}

\section{Plant material and sample preparation}

Pea (Pisum sativum var. Charmette) was grown in plant soil for about two weeks in a light/dark regime with $16 \mathrm{~h}$ light/ $8 \mathrm{~h}$ dark; $80 \mu \mathrm{mol}$ photons $\mathrm{m}^{-2} \mathrm{~s}^{-1}$ of photosynthetically active radiation. Isolation of thylakoid membranes was started with the grinding of harvested leaves in a blender in the presence of a grinding buffer containing $50 \mathrm{mM}$ HEPES (pH 7.5), $0.4 \mathrm{M} \mathrm{NaCl}, 2 \mathrm{mM}$ $\mathrm{MgCl}_{2}, 1 \mathrm{mM}$ EDTA, and $2 \mathrm{mg} \mathrm{ml}^{-1}$ BSA. Ground leaves were pressed through a cheese cloth to remove the larger pieces of plant material and the obtained solution was centrifuged at $5000 \mathrm{~g}$ for $10 \mathrm{~min}$. The pellet was resuspended in a washing buffer containing $50 \mathrm{mM}$ HEPES ( $\mathrm{pH} 7.5$ ), $0.15 \mathrm{M} \mathrm{NaCl}$, and $4 \mathrm{mM}$ $\mathrm{MgCl}_{2}$ and after centrifugation $(8000 \mathrm{~g}, 10 \mathrm{~min})$ the washing step was repeated. Finally, the pellet was resuspended in $20 \mathrm{mM}$ Bis-Tris ( $\mathrm{pH}$ 6.5) with $5 \mathrm{mM} \mathrm{MgCl}_{2}$. The isolated thylakoid membranes were solubilized with $3 \%(\mathrm{w} / \mathrm{v})$ digitonin for $20 \mathrm{~min}$ at a final chlorophyll concentration of $1 \mathrm{mg} \mathrm{m}^{-1}$ (determined in $80 \%$ acetone ${ }^{14}$ ), followed by centrifugation in an Eppendorf table centrifuge for 15-20 min. The supernatant was filtered with a $0.45 \mu \mathrm{m}$ filter to remove unsolubilized membrane pieces and PSI complexes were purified by gel-filtration chromatography on a Superdex 200 HR 10/30 column (Pharmacia) with $20 \mathrm{mM}$ BisTris (pH 6.5), $5 \mathrm{mM} \mathrm{MgCl}_{2}$, and about $0.1 \%$ digitonin as mobile phase.

\section{Electron microscopy and image analysis}

The samples were applied to glow discharge carbon-coated copper grids and stained with 2\% uranyl acetate. Electron microscopy was performed on a Philips CM20 FEG electron microscope at a magnification of $66000 \times$. The images were digitally recorded with a Gatan $4 \mathrm{~K}$ slow scan CCD camera with a pitch of $15 \mu \mathrm{m}$ and a binning factor of 2, corresponding to a pixel size of $0.45 \mathrm{~nm}$ at the specimen level. Single particle projections were selected for single particle averaging ${ }^{15}$ with Groningen Image Processing (GRIP) software on a PC cluster. Projections were aligned by multi-reference alignment using mutual correlation functions. ${ }^{16}$ The aligned images were subjected to multivariate statistical analysis (MSA). After MSA, particles were classified and summed and class sums were used in a next cycle of multireference alignment, MSA and classification.

\section{Results}

The peak fractions from FPLC with absorption at $700 \mathrm{~nm}$ were inspected by electron microscopy (Fig. 2). EM analysis of the fraction at $7.8 \mathrm{ml}$ revealed high numbers of oval-shaped particle projections, which were assigned to PSI monomers according to shape and size. Further careful examination of EM pictures revealed projections of dimeric and trimeric PSI complexes. As an example, a gallery of selected PSI trimers is shown in Fig. 3(A)-(D). The total yield of both PSI dimers and trimers was rather low, about $1.5 \%$ for each, compared to the numbers of PSI monomers. Finally, two sets of about 1700 PSI dimeric and trimeric projections were selected from approximately 4000 recorded images. Additional selection of monomeric PSI projections resulted in a data set of 10000 single particles, which was subjected to image analysis.

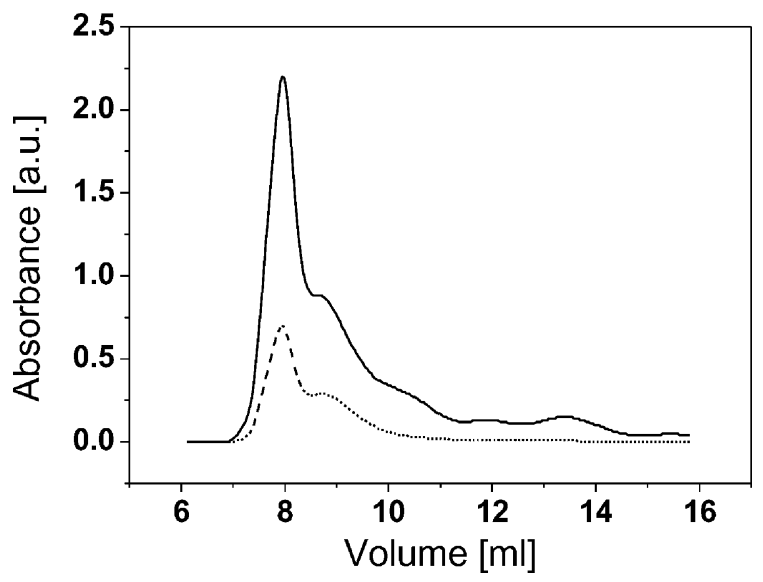

Fig. 2 FPLC gel filtration chromatogram (full line) recorded at $280 \mathrm{~nm}$ of pea thylakoid membranes solubilized with digitonin. The dotted line indicates the absorption at $700 \mathrm{~nm}$, indicative of PSI. The fraction at $7.8 \mathrm{ml}$ was used for EM studies.

Fig. 3(G) represents an averaged projection of the pea PSI monomer with similar dimensions and densities, to those previously mentioned in spinach PSI particle (Fig. 1(D)). Image analysis of trimeric complexes resulted in a classification into two main specific classes. First, Fig. 3(E) shows a class of trimeric PSI with relatively well resolved features of the core parts of the upper two PSI monomers. The features of the third monomer are less dense and blurred, which might indicate a different handedness (up-side down) and a non-specific binding 


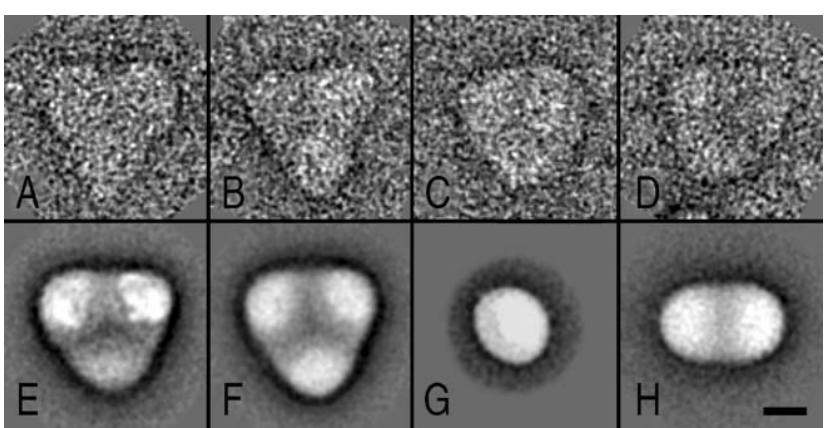

Fig. 3 (A-D) a gallery of top view projections of a PSI trimer selected from electron micrographs. (E)-(H) Classification of 10000 single particle projections. Averaged images represent sums of 290 (E), $600(\mathrm{~F}), 560(\mathrm{G})$ and $420(\mathrm{H})$ aligned projections. (E) Artificial trimer consisting of two up- and down-oriented monomers connected by part of the LHCI and of the third non-specifically associated monomer. (F), (H) Trimer and dimer, respectively, consisting of monomers associated by their LHCI complexes. (G) Monomeric PSI. The space bar equals $10 \mathrm{~nm}$.

of this monomer in the trimeric complex. Secondly, Fig. 3(F) shows a PSI trimer class without clearly discernible features in all monomers. The main mass of each monomer has a much higher stain-excluding density than the LHCI antenna and hence corresponds to the PSI core complex moiety. Since the core parts are located at the edge of the trimer, association between monomers must occur via the LHCI subunits. Image analysis of PSI dimers did not result in any predominant view of the dimeric complex due to a heterogeneity of the data set. One of resolved classes is shown in Fig. 3(H), as an example. Features of the monomers are not well resolved, however, as in the trimeric complexes, the core parts of PSI are again localized at the periphery of the dimer.

\section{Discussion}

Both the previous and the presented results show that PSI has a strong ability to associate once it is isolated from the thylakoid membrane. Different artificial dimers, trimers, tetramers, and even two-dimensional sheets were observed in preparations from spinach using $\alpha$-D-dodecyl maltoside (Fig. 1(A)-(H), (Q)). Artificial association of PSI seems to be plant non-specific, as similar artificial dimers were also found in preparations from Arabidopsis thaliana (Fig. 1(M)-(P)). Nevertheless, the association is not highly specific, since the PSI complexes do not have the same orientation with respect to the membrane anymore.

EM analysis of digitonin solubilized PSI particles also revealed the presence of dimers and trimers in the preparation. Surprisingly, the association of PSI was different compared to $\alpha$-D-dodecyl maltoside preparation (compare Figs. 3 and 1). Whereas the association between PSI monomers in $\alpha$-D-dodecyl maltoside preparation of spinach and Arabidopsis dimers is mediated mainly by a contact between the core complexes, in the case of digitonin preparation the PSI association is mediated by LHCI, which is bound at the PsaF/J side of the core complex. However, the best resolved PSI trimer (Fig. 3(E)) shows a mirrored density in the two upper monomers indicating artificial association of up- and down-oriented monomers and a lower monomer with a much lower and fuzzy density. In a native trimer, all monomers should face with the same side up in the thylakoid membrane, and thus the main densities in averaged EM projections should be related by rotational symmetry. Image analysis of the rest of the data set did not reveal any significant features in the trimer or in the dimer (Fig. 3(F), $(\mathrm{H})$, respectively). Fuzziness in the averaged projections can be explained by the non-specific or flexible association of the PSI monomers. A similar fuzziness was recently observed in some of the associations of PSI and IsiA proteins. ${ }^{17}$ From the very low number of observed dimers and trimers and from the non-specific binding of monomers in these multimers, we conclude that the green plant PSI is monomeric, although the possibility can not be excluded that a very small number of particles associate with their LHCI complexes. Whether this could mean that excitation energy transfer between such complexes would be possible is an open question. But on the scale of the complete chloroplast, it is unlikely to be of great significance.

In general our results indicate that high-molecular bands detected on blue-native gel electrophoresis, like the PSI dimers and trimers, ${ }^{11}$ can not be regarded as proof for the existence of native particles in the membrane. Another claim for a supercomplex found by this technique is the dimeric chloroplast ATP synthase in Chlamydomonas reinhardtii. ${ }^{18}$ Analysis of ATP synthase complexes within spinach thylakoid membranes, which did not undergo detergent treatment, showed only some non-specific association. ${ }^{1}$ On the other hand blue-native gel electrophoresis is a useful pointer to such complexes and it now seems likely that the PSI-LHCII particle seen on blue-native gels is a specific complex. ${ }^{19}$

\section{Acknowledgements}

We thank Dr J. P. Dekker and Prof. H. P. Braun for discussion. R. K. was supported by the European Union, grant HPRNCT-2002-00248 ("PSICO network"). The work of E. J. B. was supported by a grant from the Foundation of Life and Earth Sciences (ALW) of NWO.

\section{References}

1 J. P. Dekker and E. J. Boekema, Supramolecular organization of thylakoid membrane proteins in green plants, Biochim. Biophys. Acta, 2005, 1706, 12-39.

2 P. Jordan, P. Fromme, H. T. Witt, O. Klukas, W. Saenger and N. Krauss, Three-dimensional structure of photosystem I at $2.5 \AA$ resolution, Nature, 2001, 411, 909-917.

3 A. Ben-Shem, F. Frolow and N. Nelson, Crystal structure of plant photosystem I, Nature, 2003, 426, 630-635.

4 E. J. Boekema, P. E. Jensen, E. Schlodder, J. F. L. van Breemen, H. van Roon, H. V. Scheller and J. P. Dekker, Green plant photosystem I binds light-harvesting complex I on one side of the complex, Biochemistry, 2001, 40, 1029-1036.

5 C. Lunde, P. E. Jensen, A. Haldrup, J. Knoetzel and H. V. Scheller, The PSI-H subunit of photosystem I is essential for state transitions in plant photosynthesis, Nature, 2000, 408, 613-615.

$6 \mathrm{~S}$. Zhang and H. V. Scheller, Light-harvesting complex II binds to several small subunits of photosystem I, J. Biol. Chem., 2004, 279, 3180-3187.

7 E. J. Boekema, J. P. Dekker, M. van Heel, M. Rögner, W. Saenger, I. Witt and H. T. Witt, Evidence for a trimeric organization of the photosystem I complex from the thermophilic cyanobacterium Synechococcus sp., FEBS Lett., 1987, 217, 283-286.

8 V. P. Chitnis and P. R. Chitnis, PsaL subunit is required for the formation of photosystem I trimers in the cyanobacterium Synechocystis sp. PCC 6803, FEBS Lett., 1993, 336, 330 334.

9 I. Domonkos, P. Malec, A. Sallai, L. Kovacs, K. Itoh, G. Shen, B. Ughy, B. Bogos, I. Sakurai, M. Kis, K. Strzalka, H. Wada, S. Itoh, T. Farkas and Z. Gombos, Phosphatidylglycerol is essential for oligomerization of photosystem I reaction center, Plant Physiol., 2004, 134, 1471-1478.

10 A. Ben-Shem, F. Frolow and N. Nelson, Evolution of photosystem I-from symmetry through pseudosymmetry to asymmetry, FEBS Lett., 2004, 564, 274-280.

11 J. Heinemeyer, H. Eubel, D. Wehmhoner, L. Jansch and H. P. Braun, Proteomic approach to characterize the supramolecular organization of photosystems in higher plants, Phytochemistry, 2004, 65, 16831692 .

12 N. V. Dudkina, H. Eubel, W. Keegstra, E. J. Boekema and H. P. Braun, Structure of a mitochondrial supercomplex formed by respiratory chain complexes I and III, Proc. Natl. Acad. Sci. USA, 2005, 102, 3225-3229. 
13 E. J. Boekema, J. F. L. van Breemen, H. van Roon and J. P. Dekker, Arrangement of photosystem II supercomplexes in crystalline macrodomains within the thylakoid membrane of green plant chloroplasts, J. Mol. Biol., 2000, 301, 1123-1133.

14 R. J. Porra, W. A. Thompson and P. E. Kriedemann, Determination of accurate extinction coefficients and simultaneous-equations for assaying chlorophyll-a and chlorophyll-b extracted with 4 different solvents-verification of the concentration of chlorophyll standards by atomic-absorption spectroscopy, Biochim. Biophys. Acta, 1989, 975, 384-394.

15 G. Harauz, E. J. Boekema, E. and M. van Heel, Statistical image analysis of electron micrographs of ribosomal subunits, Methods Enzymol., 1988, 164, 35-49.

16 M. van Heel, M. Schatz and E. Orlova, Correlation functions revised, Ultramicroscopy, 1992, 46, 307-316.
17 N. Yeremenko, R. Kouřil, J. A. Ihalainen, S. D'Haene, N. van Oosterwijk, E. G. Andrizhiyevskaya, W. Keegstra, H. L. Dekker, M. Hagemann, E. J. Boekema, H. C. P. Matthijs and J. P. Dekker, Supramolecular organization and dual function of the IsiA chlorophyll-binding protein in cyanobacteria, Biochemistry, 2004, 43, 10308-10313.

18 S. Rexroth, J. M. Meyer zu Tittingdorf, H. J. Schwassmann, F. Krause, H. Seelert and N. A. Dencher, Dimeric $\mathrm{H}^{+}$-ATP synthase in the chloroplast of Chlamydomonas reinhardtii., Biochim. Biophys. Acta, 2004, 1658, 202-211.

19 R. Kouřil, A. Zygadlo, A. A. Arteni, C. D. de Wit, J. P. Dekker, P. E. Jensen, H. V. Scheller and E. J. Boekema, Structural characterization of a complex of photosystem I and light-harvesting complex II of Arabidopsis thaliana, Biochemistry, in press. 